\title{
Prospects for expanding the use of $\beta$-lactamase inhibitors
}

Since the introduction of antibiotics there has been a constant battle to develop new antimicrobial agents to combat increasing resistance to the older drugs. This has been particularly so for the $\beta$-lactam agents, which are among the most widely used antimicrobial agents worldwide. Resistance varies geographically but is generally much higher in the developing world. The most important mechanism by which bacteria become resistant to these agents is the production of $\beta$-lactamases which catalyse the hydrolysis of the $\beta$-lactam ring. Of the $\beta$-lactamases encountered in gram-negative bacilli the TEM-1 enzyme is probably the most important clinically; it can be present in up to $50 \%$ of Escherichia coli isolates and up to $85 \%$ of ampicillin-resistant $E$. coli. ${ }^{1,2}$ Recently the TEM-1 enzyme was detected in $42 \%$ of commensal $E$. coli isolates from the faeces of healthy volunteers in Scotland (Philippa Shanahan, personal communication).

Two strategies have been employed to overcome resistance mediated by $\beta$-lactamases. Firstly, the development of new $\beta$-lactam antibiotics which, as a result of modifications in structure, are not hydrolysed by most $\beta$-lactamases found amongst clinical isolates. Secondly, the use of $\beta$-lactamase inhibitors which when co-administered prevent inactivation by $\beta$-lactamases. ${ }^{3,4}$

The first strategy has yielded successive generations of $\beta$-lactams, in particular the penicillinase-resistant penicillins and the cephalosporins. To overcome resistance in gram-negative bacilli, the $\beta$-lactamasestable "second generation" and extended-spectrum "third generation" cephalosporins were developed. ${ }^{5}$ However, shortly after the introduction of these agents, plasmid-mediated resistance arose as a result of simple mutations of the already prevalent plasmid $\beta$ lactamases, especially TEM-1.4,6 More than 30 extended-spectrum $\beta$-lactamases have been identified worldwide although it is by no means certain that all are distinct. ${ }^{6}$ Reassuringly, even when these enzymes appear during treatment they rarely persist and they have not yet displaced TEM-1 as the most prevalent $\beta$-lactamase. ${ }^{6}$ There have been occasional reports of extended-spectrum $\beta$-lactamases, in particular TEM3 , appearing with high frequency but these have been local outbreaks probably related to spread of the host strain. $^{7}$ Why these $\beta$-lactamases have not spread further is unclear but the mutation allowing hydrolysis of the extended-spectrum cephalosporins may render the enzymes less efficient against simple penicillins which remain the major selective pressure-a situation which may change with the recent introduction of oral preparations of extended-spectrum cephalosporins.

The problem of extended-spectrum $\beta$-lactamases has led to a renewed interest in $\beta$-lactamase inhibitors, particularly as all the TEM-derived and SHV-derived extended-spectrum $\beta$-lactamases identified to date are inhibited by clavulanic acid, sulbactam and tazobactam. ${ }^{8,9}$ There have been two recent reports of resistance to expanded-spectrum cephalosporins caused by plasmid-mediated $\beta$-lactamases that were not inhibited by clavulanic acid; these enzymes, designated BIL-1 and MIR-1, are not related to TEM but appear to be chromosomal $\beta$-lactamases which have migrated on to plasmids. ${ }^{10,11}$

Despite widespread use within hospitals and in general practice, resistance to co-amoxiclav (amoxycillin plus clavulanic acid) remains low $^{12}$ although there have been reports of resistance resulting from hyperproduction of the TEM- $1 \beta$-lactamase. ${ }^{13,14}$ The exact mechanism is unclear but several possibilities have been proposed, including plasmid rearrangement to give small multicopy plasmids and gene duplication. ${ }^{15,16}$ It is not known whether such strains arose as a result of use of co-amoxiclav or already existed in the bacterial population before the introduction of inhibitor combinations. ${ }^{2,17}$ Although strains producing higher levels of TEM-1 require more clavulanic acid to potentiate amoxycillin, there has been no increase in the frequency of such strains in clinical isolates between 1982 and $1989 .{ }^{2}$

It has been argued that mutation to clavulanic acid resistance in the TEM-1 $\beta$-lactamase would deprive the enzyme of its catalytic ability to hydrolyse $\beta$ lactam antibiotics. However, such enzymes have been selected in vitro and have recently been detected in clinical isolates. ${ }^{18-20}$ They have a lower $\mathrm{pI}$ than TEM1 and require a higher concentration of clavulanic acid to inhibit their activity. The plasmid-encoded $\beta$ lactamase TRC-1, isolated from a clinical source, increases the MIC of co-amoxiclav for a standard $E$. coli recipient from 4 to $32 \mathrm{mg} / \mathrm{L}$, but results in an MIC of amoxycillin alone of only $256 \mathrm{mg} / \mathrm{L}$; the MIC of amoxycillin for TEM-1 producing strains is normally $1024 \mathrm{mg} / \mathrm{L}$. Mutant forms of the TEM-1 $\beta$-lactamase selected in vitro with co-amoxiclav have the same $\mathrm{pI}$ as TRC-1 and a similarly raised resistance to inhibition by clavulanic acid; the MIC of amoxycillin for strains harbouring this enzyme are reduced from 1024 to $128 \mathrm{mg} / \mathrm{L}$ (Thomson and Amyes, unpublished results). It is unclear if such enzymes will have any significant clinical impact, but after ten years of use of 
inhibitor combinations they still appear to be rare. Furthermore, the increased resistance to clavulanic acid may not compensate for the reduced activity against amoxycillin.

The most commonly prescribed $\beta$-lactamase inhibitor has been clavulanic acid in combination with ticarcillin or amoxycillin. Sulbactam, a penicillanic acid sulphone, is now available in combination with ampicillin or, in some countries, with cefoperazone. The related sulphone, tazobactam, shows in-vitro activity comparable with that of clavulanic acid. If new $\beta$-lactamase inhibitors are developed, how will they be used? At present they are available only in fixed combination with $\beta$-lactam agents in the UK, but if the newer expanded-spectrum $\beta$-lactamases become

\section{References}

1. Thomson CJ, Hood J, Miles RS, Amyes SGB. Prevalence of the TEM-1 $\beta$-lactamase in urinary $E$. coli: continued efficacy of amoxycillin plus clavulanic acid. Abstracts of the 1st International Congress on the Management of Infection No.P1.21. 1992: 73.

2. Seetulsingh PS, Hall LMC, Livermore DM. Activity of clavulanate combinations against TEM- $\beta$-lactamase producing Escherichia coli isolates obtained in 1982 and 1989. $J$ Antimicrob Chemother 1991; 27: 749-759.

3. Rolinson GN. Evolution of $\beta$-lactamase inhibitors. Rev Infect Dis 1991; 13 Suppl 9: S727-S732.

4. Philippon A, Labia R, Jacoby G. Extended-spectrum $\beta$ lactamases. Antimicrob Agents Chemother 1989; 33: 1131-1136.

5. Wiedemann B, Kliebe C, Kresken $\mathbf{M}$. The epidemiology of $\beta$ lactamases. J Antimicrob Chemother 1989; 24 Suppl B: $1-22$.

6. Payne DJ, Amyes SGB. Transferable resistance to extended spectrum $\beta$-lactams: a major threat of a minor inconvenience? J Antimicrob Chemother 1991; 27: 255-261.

7. Sirot J, Chanal C, Petit A, Sirot D, Labia R, Gerbaud G. Klebsiella pneumoniae and other Enterobacteriaceae producing novel plasmid-mediated $\beta$-lactamases markedly active against third-generation cephalosporins: epidemiological studies. Rev Infect Dis 1988; 10: 850-859.

8. Payne DJ, Cramp R. Determination of $\mathrm{ID}_{50}$ values for 35 plasmid mediated $\beta$-lactamases. Abstracts of the 1st International Congress on the Management of Infection No. 10B.7. 1992: 65 .

9. Jarlier V, Nicolas MH, Fournier G, Philippon A. Extended broad spectrum $\beta$-lactamases conferring transferable resistance to newer $\beta$-lactam agents in Enterobacteriaceae: hospital prevalence and susceptibility patterns. Rev Infect Dis $1988 ; 10$ : 867-878.

10. Payne DJ, Woodford N, Amyes SGB. Characterization of the plasmid $\beta$-lactamase BIL-1. J Antimicrob Chemother 1992; 30: $119-127$.

11. Papanicolaou GA, Medeiros AA, Jacoby GA. Novel plasmid- a clinical problem, there may be a case for using them in combination with aminothiazole cephalosporins, or making them available as individual agents, leaving the final choice of antibiotic partner to the clinician, as currently occurs in some countries.

The use of $\beta$-lactamase inhibitors is an important strategy in the fight against resistance to $\beta$-lactam antibiotics. Increased use may lead to resistance, but the fact that the inhibitor binds to the active site of the $\beta$-lactamase suggests that $\dot{\beta}$-lactamase-mediated resistance may be of lesser importance.

\section{J. Thomson and S. G. B. Amyes}

Department of Medical Microbiology, The Medical School, University of Edinburgh, Teviot Place, Edinburgh EH8 9AG

mediated $\beta$-lactamase (MIR-1) conferring resistance to oxyimino- and -mthoxy $\beta$-lactams in clinical isolates of Klebsiella pneumoniae. Antimicrob Agents Chemother 1990; 34: 2200-2209.

12. Rubio MC, Gil J, Castillo $\mathrm{J}$ et al. The susceptibility to amoxycillin/clavulanate of Enterobacteriaceae with plasmid-mediated ampicillin resistance: a twelve-year study of strains in one Spanish hospital. J Antimicrob Chemother 1989; 24 Suppl B: $35-40$

13. Sanders CC, Iaconis JP, Bodey GP, Samonis G. Resistance to ticarcillin-potassium clavulanate among clinical isolates of the family Enterobacteriaceae: Role of PSE- $1 \beta$-lactamase and high levels of TEM-1 and SHV-1 and problems with false susceptibility in disk diffusion tests. Antimicrob Agents Chemother 1988; 32: 1365-1369.

14. French G, Ling J. Amoxycillin/clavulanate resistant Escherichia coli. Lancet 1988; $1: 704$.

15. Shannon K, Williams H, King A, Phillips I. Hyperproduction of TEM-1 $\beta$-lactamase in clinical isolates of Escherichia coli serotype O15. FEMS Microbiol Lett 1990; 55: 319-323.

16. Martinez JL, Vicente MF, Delgado-Iribarren A, Perez-Diaz JC, Baquero F. Small plasmids are involved in amoxicillinclavulanate resistance in Escherichia coli. Antimicrob Agents Chemother 1989; 33: 595.

17. Page JWJ, Farmer TH, Elson SW. Hyperproduction of TEM-1 $\beta$-lactamase by Escherichia coli strains. J Antimicrob Chemother 1989; 23: 160-161.

18. Thomson CJ, Amyes SGB. TRC-1: Emergence of a clavulanic acid-resistant TEM $\beta$-lactamase in a clinical strain. FEMS Microbiol Lett 1992; 91: 113-118.

19. Belaaouaj A, Lapoumeroulie C, Vedel G, Nevot P, Krishnamoorthy R, Paul G. Amino acid 241 of TEM-1 is highly critical in conferring resistance to ampicillinclavulanic acid combination: molecular characterisation of a natural mutant. Abstracts of the 31st I.C.A.A.C. No. 944. 1991: 256

20. Manavathu EK, Lerner SA, Fekete T, Perlin MH, Ziaja E, Price $S$. Characterization of mutant TEM $\beta$-lactamase that confers resistance to ampicillin (amp) plus clavulanic acid (CA). Abstracts of the 30th I.C.A.A.C. No. 281. 1990: 133. 